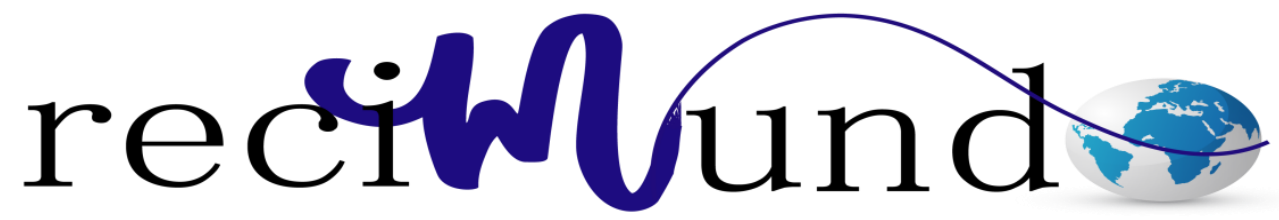

Revista Científica Mundo de la Investigación y el Conocimiento

Letty Teresa Parrales Pinargote a ; Edwin Leonardo Gavilánez Macías ${ }^{\text {b }}$; Alba Amarilis Sornoza Pin ${ }^{c}$; Silvia Verónica Amen Carrillo ${ }^{\text {; }}$ Carlos Alberto Riofrio Pinargote $^{\text {e }}$, Noralma Katherine Jaime Hernández ${ }^{\mathrm{f}}$

Aspectos clínicos y epidemiológicos de los casos de zika en mujeres embarazadas del Cantón Jipijapa

Revista Científica Mundo de la Investigación y el Conocimiento. Vol. 2 núm.2, julio, ISSN: 2588-073X, 2018, pp. 782-793

DOI: $10.26820 /$ recimundo/2.(2).2018.782-793

Editorial Saberes del Conocimiento

Recibido: 05/12/2017 Aceptado: $15 / 03 / 2018$

a. Distrito 13D03 Jipijapa - Puerto López; Lic. Enfermería "Centro de Salud Jipijapa"

b. Médico tratante "Centro de Salud Puerto López" Distrito de Salud 13D03 Jipijapa - Puerto López

c. Facultad de Enfermería, Universidad Laica Eloy Alfaro de Manabí. Manta - Manabí - Ecuador.

d. Médico Tratante "Centro de Salud América" Distrito de Salud 13D03 Jipijapa - Puerto López.

e. Distrito 22D02 Orellana - Loreto - Salud Lic. Enfermería "Parroquia García Moreno"

f. Carrera de Enfermería, Facultad Ciencias de la Salud, Universidad Estatal del Sur de Manabí. Campus Los Ángeles Km.1 vía a Noboa. Jipijapa - Manabí - Ecuador; norahernandez10@ hotmail.es 


\section{Aspectos clínicos y epidemiológicos de los casos de zika en mujeres embarazadas del Cantón Jipijapa}

Vol. 2, núm. 2., (2018)

Letty Teresa Parrales Pinargote; Edwin Leonardo Gavilánez Macías; Alba Amarilis Sornoza Pin;

Silvia Verónica Amen Carrillo; Carlos Alberto Riofrio Pinargote; Noralma Katherine Jaime

Hernández

\section{RESUMEN}

El zika es una enfermedad infecciosa causada por el vector Aedes aegypti que de presentarse en las gestantes podrían existir daños neurológicos en el feto, el objetivo de esta investigación es determinar los aspectos clínicos y epidemiológicos de los casos positivos y sospechoso de zika en mujeres embarazada durante el periodo enero-diciembre 2016 en el Hospital Básico Jipijapa, se realizó un estudio retrospectivo, descriptivo y cualitativo, para obtener la información sobre la investigación, se aplicó la técnica de encuesta realizada a las 22 gestantes ingresadas en el sistema de vigilancia epidemiológica, los principales resultados revelaron que las madres adquirieron el virus del zika durante el último trimestre, tiempo en el que las complicaciones son mínimas a diferencia del primer trimestre de gestación, encontrándose la mayor parte de las afectadas en las zonas urbano marginales.

Palabras claves: Virus, Aedes aegypti, cadena epidemiológica, enfermedades, epidemiologia, morbilidad. 


\title{
Aspectos clínicos y epidemiológicos de los casos de zika en mujeres embarazadas del Cantón Jipijapa
}

Vol. 2, núm. 2., (2018)

Letty Teresa Parrales Pinargote; Edwin Leonardo Gavilánez Macías; Alba Amarilis Sornoza Pin; Silvia Verónica Amen Carrillo; Carlos Alberto Riofrio Pinargote; Noralma Katherine Jaime Hernández

\begin{abstract}
Vectors are living organisms that can transmit infectious diseases such as the Aedes Aegypti mosquito transmitting the Zika virus and if present in pregnant women there could be neurological damage in the fetus, the objective of this research is to determine the clinical and epidemiological aspects of positive cases And suspected of zika in pregnant women during the period January-December 2016 at the Jipijapa Basic Hospital, a retrospective, descriptive and qualitative study was carried out to obtain the information about the investigation, the survey technique applied to the 22 pregnant women admitted In the epidemiological surveillance system, the main results revealed that the mothers acquired the zika virus during the last trimester, at which time the complications were minimal, as opposed to the first trimester of gestation, with most of those affected in urban areas therefore.
\end{abstract}

Keywords: Virus, Aedes aegypti, epidemiological chain, diseases, epidemiology, morbidity. 


\section{Aspectos clínicos y epidemiológicos de los casos de zika en mujeres embarazadas del Cantón Jipijapa}

Vol. 2, núm. 2., (2018)

Letty Teresa Parrales Pinargote; Edwin Leonardo Gavilánez Macías; Alba Amarilis Sornoza Pin;

Silvia Verónica Amen Carrillo; Carlos Alberto Riofrio Pinargote; Noralma Katherine Jaime

Hernández

\section{Introducción.}

El virus del zika es un flavivirus transmitidos por mosquitos que se identificó por primera vez en macacos, a través de una red de monitoreo de la fiebre amarilla. Posteriormente, en 1952, se identificó en el ser humano en Uganda y la republica unida de Tanzania, se han registrado brotes de enfermedad por este virus en África, las Américas, Asia y el Pacifico. Hace casi 70 años varios científicos descubrieron el virus que hoy tiene en alerta al mundo, lo hallaron en monos que eran monitoreados científicamente para estudios de la fiebre amarilla, siete años antes de que fuera detectada la primera infección en humanos, siendo el Aedes aegypti, el principal vector de la epidemia por lo que el virus del zika está proyectado como una de las enfermedades que emergen en el siglo XXI , “ la Organización Mundial de la Salud declaro el 1 de febrero del 2016 emergencia sanitaria global y así realizar un arduo trabajo para evitar o reducir los efectos graves de esta epidemia (1)".

Ante la introducción de este nuevo virus y su asociación con la microcefalia entre los hijos de madres infectadas y a otras alteraciones neurológicas como el Síndrome de guillen barre, ha sido centro de firme estudio descubriéndose cada día nuevos hallazgos referente a dicha enfermedad. Las embarazadas tienen el mismo riesgo que el resto de la población de infectarse con el virus del Zika. "Además, la infección materna por el virus de Zika puede pasar desapercibida, ya que muchas personas no presentan síntomas. Aunque los síntomas que causa esta infección durante el embarazo, cuando aparecen, suelen ser leves" (2). De forma característica zika causa infección asintomática correspondiendo a un $80 \%$ de los casos en la que 


\section{Aspectos clínicos y epidemiológicos de los casos de zika en mujeres}

embarazadas del Cantón Jipijapa

Vol. 2, núm. 2., (2018)

Letty Teresa Parrales Pinargote; Edwin Leonardo Gavilánez Macías; Alba Amarilis Sornoza Pin; Silvia Verónica Amen Carrillo; Carlos Alberto Riofrio Pinargote; Noralma Katherine Jaime Hernández

los afectados no presentan mayor sintomatología y un $20 \%$ que los presentan, la conjuntivitis no purulenta lo que caracteriza la infección del virus o puede presentarse signos y síntomas parecidos al Dengue o el Chicungunya pero de una forma más leve, por lo que esto conlleva a que no se trabaje activamente en la detección y confirmación de casos de zika, especialmente en las embarazadas y así poder efectuar tácticas efectivas que minimicen la presencia del mosquito y por ende evitar que el producto de la gestante se vea afectado, que de acorde a las investigaciones es donde hay mayor repercusión. Los principales factores para una transmisión es la susceptibilidad de los humanos en adquirir el virus y el desconocimiento de la misma, por lo tanto es necesario hacer énfasis en la difusión y promoción sobre el virus del Zika y toda su epidemiologia.

De acuerdo a las diferentes investigaciones existe evidencia que en 61 países y territorios del mundo donde existe la presencia del virus de las cuales América es el continente que más casos ha reportado, siendo Brasil y Colombia los más afectados. "El brote de la enfermedad del virus del zika en la región de las Américas de la Organización Mundial de la Salud comenzó en Brasil, donde se registró el primer brote del virus del Zika confirmado en laboratorio, en mayo del 2015; Colombia confirmo la transmisión local del virus del Zika casi 5 meses más tarde, en octubre del 2015” (3). Anteriormente el brote del virus en Brasil no originó alarma hasta que se descubrió un aumento de casos de microcefalia en niños nacidos de madres que vivían en zonas donde el virus había sido identificado, luego la aparición de nuevos casos en los diferentes países en la que confirmaron la relación que existía entre el virus del zika y las malformaciones congénitas y las pruebas de que el virus además de que se pueda transmitir de la madre al feto a 


\section{Aspectos clínicos y epidemiológicos de los casos de zika en mujeres embarazadas del Cantón Jipijapa}

Vol. 2, núm. 2., (2018)

Letty Teresa Parrales Pinargote; Edwin Leonardo Gavilánez Macías; Alba Amarilis Sornoza Pin;

Silvia Verónica Amen Carrillo; Carlos Alberto Riofrio Pinargote; Noralma Katherine Jaime

Hernández

lo largo del embarazo, se transmite a través de vía sanguínea y sexual lo que atrajo la atención de los medios de comunicación y así contribuir a estudiar las hipótesis en torno al virus ya que como se mencionó anteriormente la población se enfrente a uno de los mayores desafíos en cuanto a este brote del virus donde el desconocimiento de la magnitud del riesgo y los resultados asociados a la enfermedad durante el embarazo.

El virus y sus efectos, impresionan de manera alarmante a Colombia siendo esté el segundo país más afectado. "En Colombia, entre la semana epidemiológica 32 del 2015 a la semana 45 del 2016 (9 de agosto del 2015 y el 12 de noviembre del 2016) se registraron 105.372 casos los cuales han sido diagnosticados según manifestaciones clínicas, con o sin confirmación de exámenes de laboratorio, incluyendo 19443 casos confirmados y sospechosos en mujeres gestantes" (4). De acuerdo a los investigaciones se registraron mayores casos de zika en mujeres que en hombres, y los casos reportados a el sistema de vigilancia con síndromes neurológicos relacionados a la infección del virus fue de 659 y 58 casos de microcefalia, así este es un hecho relevante y por primera vez descrito en la historia. Colombia reporta la transmisión del virus en dicho país el 16 de octubre del 2015.

La enfermedad temida por muchos al afectar a Latinoamérica también afecta a Ecuador reportando el primer caso de zika el 20 de octubre del 2015, cuatro días después del reporte de Colombia, exponiendo alerta epidemiológica. "Hasta la semana epidemiológica 1-5 del 2017 se habían reportado 3008 casos confirmados en Ecuador, 933 casos confirmados por laboratorio y 2073 casos por nexo epidemiológico" (5). 
Aspectos clínicos y epidemiológicos de los casos de zika en mujeres embarazadas del Cantón Jipijapa

Vol. 2, núm. 2., (2018)

Letty Teresa Parrales Pinargote; Edwin Leonardo Gavilánez Macías; Alba Amarilis Sornoza Pin; Silvia Verónica Amen Carrillo; Carlos Alberto Riofrio Pinargote; Noralma Katherine Jaime Hernández

De acuerdo a las cifras reportadas la última semana epidemiológica del año 2015 y durante el año 2016 fueron 2942 casos reportados a nivel nacional Manabí es la provincia que represento mayor número de casos con un 85,2\%, seguido de Esmeraldas con un total de 191 casos y la provincia del guayas ocupando un tercer lugar con una cifra de 112 casos. De acuerdo a las investigaciones la población femenina fue la más afectada en edades comprendidas entre 20 a 49 años, de las cuales se notificaron 239 casos confirmadas de zika en embarazadas, las edades gestacionales de estas mujeres comprendían 61 casos menor a 12 semanas, 113 mayor a 12 y 65 casos mayor a 28 semanas. En lo que lleva del año 2017 son 64 casos confirmados, de ellos 54 por laboratorio y 10 por nexo epidemiológico lo que demuestra de estos nuevos brotes es la potencialidad de este arbovirus para propagarse. (6-9)

Dada la presencia de este mosquito transmisor, lo que ante la situación que origina la provincia de Manabí con 2.523 casos de zika sea una de las más afectadas, se siga mejorando los esfuerzos para implementar estrategias que ayuden a una buena comunicación con la población para así reducir la densidad del vector las cuales se relacionan con la vigilancia, el manejo clínico y las medidas de prevención y control.

\section{Material y métodos.}

El objetivo principal de la investigación es determinar los aspectos clínicos y epidemiológicos de los casos confirmados y sospechosos de zika en embarazadas del Hospital Básico Jipijapa, conociendo así cuáles son los principales problemas que afectan a esta población, se iniciará esta investigación con técnicas de recolección de datos los cuales nos 


\section{Aspectos clínicos y epidemiológicos de los casos de zika en mujeres embarazadas del Cantón Jipijapa}

Vol. 2, núm. 2., (2018)

Letty Teresa Parrales Pinargote; Edwin Leonardo Gavilánez Macías; Alba Amarilis Sornoza Pin;

Silvia Verónica Amen Carrillo; Carlos Alberto Riofrio Pinargote; Noralma Katherine Jaime

Hernández

facilitaran la descripción de datos y características de la población seleccionada, permitiendo la adquisición de datos objetivos, precisos y sistemáticos.

La población de la presente investigación está conformada por las 22 gestantes que presentaron diagnóstico de zika positivo y sospechoso en el Hospital Básico Jipijapa, no se aplicó la técnica de muestreo por el número de casos presentados. Dentro de los criterios de inclusión fue toda población de estudio, no hubo criterios de exclusión.

La investigación es de tipo retrospectiva, descriptiva y transversal, se realizó encuesta a las pacientes que presentaron diagnóstico de Zika positivo y sospechoso en el Hospital Básico Jipijapa en el periodo Enero-Diciembre del 2016. La variable independiente del proyecto de investigación son los aspectos clínicos y epidemiológicos de los casos de zika la cual va a influir, incidir o afectar a la variable dependiente que son las mujeres del cantón jipijapa.

Se aplicaron encuestas que fueron dirigidas a las madres gestantes con diagnostico zika positivo y sospechoso durante el año enero-diciembre 2016 del Cantón Jipijapa, mediante el cual pudimos obtener información oportuna y confiable.

\section{Resultados.}

Mediante la encuesta realizada a las 22 usuarias seleccionadas como muestra, se observó a 12 de ellas que representa el 55\% viven en zona urbana, y 10 mujeres con el $45 \%$ viven en zona urbana marginal, se evidencio que existe mayor índice de participantes por edad en los rangos de 13 a 21 años donde existe 12 mujeres que corresponden al 55\%, de 22 a 35 años de 


\section{Aspectos clínicos y epidemiológicos de los casos de zika en mujeres}

embarazadas del Cantón Jipijapa

Vol. 2, núm. 2., (2018)

Letty Teresa Parrales Pinargote; Edwin Leonardo Gavilánez Macías; Alba Amarilis Sornoza Pin; Silvia Verónica Amen Carrillo; Carlos Alberto Riofrio Pinargote; Noralma Katherine Jaime Hernández

edad se 9 mujeres con el 41\%, y en la categoría de más de 35 años existió 1 usuaria correspondiendo al $5 \%$.

Al plantear la pregunta cuantos controles se realizó durante el embarazo a monitorear su estado, la información indica que 2 usuarias se realizaron menos de 5 controles siendo un 9\%, existiendo 9 de ellas que se realizaron 5 controles prenatales evidenciando un 41\%, y 11 mujeres que se realizaron más de 5 controles con al 50\%, encontrando de esta manera que la mayor parte de la muestra de estudio se realizó más de 5 controles prenatales.

Según la encuestas el trimestre de embarazo en que se encontraban las gestantes al momento de adquirir el virus del zika da como resultado que 12 de las mujeres correspondiendo a un 55\% estaban cursando el tercer trimestre y 10 gestantes que representa el $45 \%$ se encontraban en el segundo trimestre de embarazo, demostrándose que la mayor parte de las embarazadas antes mencionada al adquirir el virus se encontraban en su tercer trimestre de embarazo.

Al realizar las encuestas para saber si las gestantes presentaron signos y síntomas antes de que le detectaran el virus del zika el $100 \%$ de ellas respondió que si presentaron síntomas o indicios que poseían el virus. Según los síntomas de las encuestadas el 68\% de ellas presentaba erupciones en la piel (15 encuestadas), mientras que 5 de ellas presentaron conjuntivitis no purulenta siendo este el $23 \%$ y mientras que 2 gestantes presentaron malestar general $9 \%$. 


\section{Aspectos clínicos y epidemiológicos de los casos de zika en mujeres embarazadas del Cantón Jipijapa}

Vol. 2, núm. 2., (2018)

Letty Teresa Parrales Pinargote; Edwin Leonardo Gavilánez Macías; Alba Amarilis Sornoza Pin;

Silvia Verónica Amen Carrillo; Carlos Alberto Riofrio Pinargote; Noralma Katherine Jaime

Hernández

Al analizar e interpretar los datos obtenidos en la pregunta sobre si presentaron alguna complicación durante el embarazo, parto o nacimiento del niño con un $100 \%$ correspondiente a las 22 mujeres indicaron no presentar complicación.

Con la presentación de la pregunta formulada para conocer si la población habría viajado 15 días antes de presentar los signos y síntomas del virus del zika se obtuvo que las 22 usuarias correspondientes al $100 \%$ no habrían viajado en los días antes mencionados.

Mediante los datos recolectados se obtuvo que con un $68 \%$ correspondiente a 15 usuarias les detectaron el virus del zika mediante exámenes de laboratorio y manifestaciones clínicas y un $23 \%$ que corresponde a 5 mujeres fueron detectadas por nexo epidemiológico o manifestaciones clínicas, mientras el 2\% manifestó que le detectaron el virus por ambas

Al realizar las encuestas para saber el tipo de tratamiento que recibió cuando le detectaron el virus del zika el 18\% de las gestantes respondieron que recibió tratamiento con loratadina, mientras que el $45 \%$ se acogió al tratamiento mediante paracetamol y el restantes que corresponde al $8 \%$ manifestó que no recibió ninguno tratamiento.

Según la pregunta si las gestantes recibieron visitas domiciliarias por parte del personal de salud se obtuvo los siguientes resultados el $73 \%$ de la población no le realizaron visitas domiciliarias y un $27 \%$ las recibió por parte del personal de salud. Lo que demuestra que no se le está realizando el seguimiento correspondiente a las gestantes zika positivo y sospechoso. 


\section{Aspectos clínicos y epidemiológicos de los casos de zika en mujeres}

embarazadas del Cantón Jipijapa

Vol. 2, núm. 2., (2018)

Letty Teresa Parrales Pinargote; Edwin Leonardo Gavilánez Macías; Alba Amarilis Sornoza Pin; Silvia Verónica Amen Carrillo; Carlos Alberto Riofrio Pinargote; Noralma Katherine Jaime Hernández

\section{Discusión.}

La enfermedad del zika es un problema que representa gran impacto en las embarazadas por los daños que se pueden presentar en el feto si se adquiere durante los primeros trimestre, de acuerdo al Sistema de Vigilancia Epidemiológica se registraron un total de 22 casos de los cuales el 55\% fueron detectados en el tercer trimestre de gestación, siendo la población adolescente más afectada, realizando pruebas diagnósticas obteniéndose el $45 \%$ con diagnóstico de zika positivo en el cual se obtuvo la importancia de prevenir la infección del virus del zika y a la vez monitorear a las madres gestantes infectadas del virus por ser una población de riesgo a contraer malformaciones en el feto.

Se analizaron los datos estadísticos de acuerdo a los controles prenatales en las gestantes con zika positivo y sospechoso, donde se obtuvo que el 50\% de las gestantes si cumplió con el número de controles considerado como mínimo por el Ministerio de Salud Pública.

Se establecieron factores de riesgo y las complicaciones que puede causar la infección del virus del zika en las embarazadas del cantón Jipijapa, según datos recolectados en el Hospital Básico y la encuesta aplicada se determinó que no presentaron complicaciones maternas, la mayor muestra de estudio son procedentes de zonas urbanos marginales donde existe acumulación de recipientes que puede almacenar o acumular agua limpia y favorece la proliferación del mosquito transmisor del virus, sea en el propio hogar y sus alrededores. 


\section{Aspectos clínicos y epidemiológicos de los casos de zika en mujeres embarazadas del Cantón Jipijapa}

Vol. 2, núm. 2., (2018)

Letty Teresa Parrales Pinargote; Edwin Leonardo Gavilánez Macías; Alba Amarilis Sornoza Pin;

Silvia Verónica Amen Carrillo; Carlos Alberto Riofrio Pinargote; Noralma Katherine Jaime

Hernández

\section{Bibliografía.}

1. OMS. enfermedad por el virus de zika. [Online].; 2016 [cited 2016 septiembre. Available from: http://www.who.int/mediacentre/factsheets/zika/es/.

2. Organizacion Mundial de la Salud. Organizacion Mundial de la Salud. [Online].; 2016 [cited 2016 marzo

2.

Available from: http://www.who.int/csr/resources/publications/zika/pregnancy-management/es/.

3. Liliana C, Tong TV, MPH2, Rozo N, Valencia D, MS2, et al. CDC (Centro para el control y la prevvencion de enfermedades). [Online].; 2016 [cited 2016 diciembre 16. Available from: https://espanol.cdc.gov/enes/mmwr/volumes/65/wr/mm6549e1.htm?mobile=nocontent.

4. Instituto Nacional de Salud. Semana epidemiologica 45 Bogotá, Colombia 2016. [Online]. [cited 2016. Available from: http://www.ins.gov.co/boletinepidemiologico/Boletn\%20Epidemiolgico/2016\%20Bolet\%C3\%ADn\%20epidemiol\%C3\%B 3gico\%20semana\%2045.pdf.

5. Ministerio de Salud Publica. Direccion Nacional de Vigilancia Epidemiologica. [Online].; 2017 [cited 2017 febrero 9. Available from: http://www.salud.gob.ec/wpcontent/uploads/2015/12/GACETA-ZIKA_SE5corregido.pdf.

6. OPS. [Online].; 2016 [cited 2016 marzo 25. Available from: http://www.paho.org/hq/index.php?option=com content\&view=article\&id=11552\&Itemid=4 1711\&lang=es.

7. Martines B, PhD1 M, Julu Bhatnagar P, M. Kelly Keating D, Luciana Silva-Flannery P, Atis Muehlenbachs MP, et al. Centros para el Control y Prenevenciones de Enfermedades. [Online].; 2016 [cited 2016 febrero 19. Available from: https://espanol.cdc.gov/enes/mmwr/volumes/65/wr/mm6506e1.htm?s_cid=mm6506e1_w\&m obile=nocontent.

8. Pacheco ES, Norambuena GM, Olivares CR, LuppMario Luppi N.(2) PGG. Virus Zika. Epidemiología, manifestaciones clínicas y prevención. Revista Hospital Clínico Universidad de Chile. 2016 junio.

9. Cabrera P. Epidemiologia del virus Zika. 2014. 